\title{
Role of MiR-3619-5p in $\beta$-Catenin- Mediated Non-Small Cell Lung Cancer Growth and Invasion
}

\author{
Xuecai Niu Sen Liu $^{\mathrm{b}}$ Li Jia ${ }^{\mathrm{a}} \quad$ Jing Chen ${ }^{\mathrm{c}}$ \\ aDepartment of Radiation Therapy, the Forth People's Hospital of Jinan, Jinan, ${ }^{\mathrm{D} D e p a r t m e n t}$ of \\ Oncology, Jinan Central Hospital Affiliated to Shandong University, Jinan, 'ICU, the Forth People's \\ Hospital of Jinan, Jinan, China
}

\section{Key Words}

Non-small cell lung cancer (NSCLC) • $\beta$-catenin - MiR-3619-5p • Bioinformatics analyses • miRNAs

\begin{abstract}
Background/Aims: The malignancy of non-small cell lung cancer (NSCLC) is largely due to its fast growth and invasion. WNT/ $\beta$-catenin signaling plays a critical role in regulating NSCLC carcinogenesis. Hence, suppression of $\beta$-catenin signal transduction in NSCLC cells may improve the therapeutic outcome. Methods: We analyzed the levels of $\beta$-catenin and miR-3619-5p in NSCLC specimens, compared to paired non-tumor normal lung tissue (NT). We did Bioinformatics analyses on the binding sites of 3'-UTR of $\beta$-catenin mRNA by miR3619-5p. We modified the levels of miR-3619-5p in NSCLC cells and examined their effects on $\beta$-catenin levels, and on the growth and invasion of NSCLC cells in an MTT assay and a transwell cell migration assay, respectively. Results: NSCLC specimens had significant higher levels of $\beta$-catenin, and significantly lower levels of miR-3619-5p, compared to NT. The levels of $\beta$-catenin and miR-3619-5p were inversely correlated in NSCLC specimens. Bioinformatics analyses showed that miR-3619-5p bound to $3^{\prime}$-UTR of $\beta$-catenin mRNA in NSCLC cells to inhibit its translation. Overexpression of miR-3619-5p decreased $\beta$-catenin protein, while depletion of miR-3619-5p increased $\beta$-catenin protein in NSCLC cells, without altering $\beta$-catenin mRNA levels. Overexpression of miR-3619-5p in NSCLC cells inhibited cell growth and invasion, while depletion of miR-3619-5p in NSCLC lines increased cell growth and invasion. Conclusion: Our data demonstrate a previously unappreciated role for miR-3619-5p in suppression of $\beta$-catenin-mediated cancer growth and invasion in NSCLC cells, and highlight miR-3619-5p as a novel cancer suppressor in NSCLC.
\end{abstract}




\section{Introduction}

Non-small cell lung cancer (NSCLC) is a prevalent cancer and contains three subtypes: squamous cell carcinoma, large cell carcinoma, and adenocarcinoma [1-4]. NSCLCs are often insensitive to chemotherapy and radiotherapy, and are fast-growing and highly invasive [310]. Thus, understanding of the mechanisms underlying the growth and invasion of NSCLC are extremely important for its therapy.

WNT signaling pathway has been suggested to play a critical role in the carcinogenesis of NSCLC [11-13]. In murine models, activation of WNT signaling is associated with NSCLC initiation, progress and invasion [11-13]. Several WNT pathway components may be overexpressed in NSCLC, including the key factor of WNT signaling, $\beta$-catenin [14]. Aberrant activation of WNT/ $\beta$-catenin has been associated with poor prognosis clinically [14]. On the other hand, downregulation of WNT signaling inhibited NSCLC cell proliferation and xenograft growth, reduced cell motility and invasion, and induced a more differentiated phenotype [15-17].

The translation of most proteins has been found to be regulated by microRNAs (miRNAs), which target the 3'-UTR of mRNA of certain genes [4, 18-25]. Among all miRNAs, the role of miR-3619-5p in cancers has not been reported so far.

Here, we showed that NSCLC specimens had significant higher levels of $\beta$-catenin, and significantly lower levels of miR-3619-5p, compared to paired non-tumor tissue. The levels of $\beta$-catenin and miR-3619-5p are inversely correlated in NSCLC specimens. Bioinformatics analyses showed that miR-3619-5p bound to 3'-UTR of $\beta$-catenin mRNA in NSCLC cells to inhibit its translation. Overexpression of miR-3619-5p decreased $\beta$-catenin protein, while depletion of miR-3619-5p increased $\beta$-catenin protein in NSCLC cells, without affecting $\beta$-catenin mRNA levels. Overexpression of miR-3619-5p in NSCLC cells inhibited cell growth and invasion, while depletion of miR-3619-5p in NSCLC lines increased cell growth and invasion.

\section{Materials and Methods}

\section{Patient specimen}

Resected cancer specimens from 25 NSCLC patients were obtained together with the matched tumoradjacent normal lung tissues (NLT) from 2009 to 2013 at the Forth People's Hospital of Jinan. All patients provided signed, informed consent for their tissues to be used for scientific research. Ethical approval for the current study was obtained from the Forth People's Hospital of Jinan. All diagnoses were based on pathological and/or cytological evidence. The histological features of the specimens were evaluated by senior pathologists according to the World Health Organization classification criteria. Tissues were obtained prior to chemotherapy and radiotherapy and were immediately frozen and stored at $-70^{\circ} \mathrm{C}$ prior to RT-qPCR and Western blot.

\section{NSCLC cell line culture}

A549 and H460 are two human NSCLC lines purchased from American Type Culture Collection (ATCC, Rockville, MD, USA), and were cultured in Dulbecco's Modified Eagle's Medium (DMEM) supplemented with 20\% fetal bovine serum (FBS, Invitrogen, Carlsbad, CA, USA) and L-glutamine in a humidified chamber with $5 \% \mathrm{CO}_{2}$ at $37^{\circ} \mathrm{C}$. The $\mathrm{A} 549$ cell line was first developed in 1972 by Dr. Giard through the removal and culturing of cancerous lung tissue in the explanted tumor of 58-year-old Caucasian male [26]. H460 cell line was deposited at the ATCC by Dr. Adi F. Gazdar [27]. They were both widely used NSCLC lines.

Modification of miR-3619-5p levels in NSCLC cell lines

MiR-3619-5p-modification-plasmids were prepared using routine methods. Briefly, the constructs for miR-3619-5p, or antisense (as)-miR-3619-5p or control null were cloned into pcDNA3.1-EGFP to generate the corresponding plasmids. The sequences were: miR-3619-5p sequence: 5'-UCAGCAGGCAGGCUGGUGCAGC-3', miR-3619-5p antisense sequence (as-miR-3619-5p): 5'-GCUGCACCAGCCUGCCUGCUGA-3', control null 


\section{Cellular Physiology Cell Physiol Biochem 2015;37:1527-1536

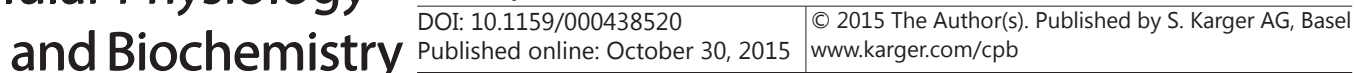 \\ Niu/Liu/Jia/Chen: MiR-3619-5p Regulates NSCLC Growth and Invasion}

sequence: 5'-UUGUACUACACAAAAGUAAUG-3'. These plasmids of $2 \mu \mathrm{g}$ were transfected into cultured NSCLC cells using Lipofectamine 2000, according to the manufacturer's instructions (Invitrogen). The plasmids also contained a GFP reporter to allow determination of transfection efficiency, which was nearly $100 \%$ in the current study.

\section{MTT assay}

For assay of cell growth, cells were seeded at $5 \times 10^{3}$ per well into a 96 well-plate and subjected to a Cell Viability Kit (MTT, Roche, Indianapolis, IN, USA), according to the instruction of the manufacturer. The MTT assay is a colorimetric assay for assessing viable cell number, taking advantage that NADPH-dependent cellular oxidoreductase enzymes in viable cells reduce the tetrazolium dye 3-(4,5-dimethylthiazol-2-yl)2,5-diphenyltetrazolium bromide (MTT) to its insoluble formazan in purple readily being quantified by absorbance value (OD) at $570 \mathrm{~nm}$ in a microtiter plate reader (Promega, Fitchburg, WI, USA). Experiments were performed 5 times.

\section{Quantitative PCR (RT-qPCR)}

MiRNA and total RNA were extracted from lung specimen or from the cultured cells with miRNeasy mini kit or RNeasy kit (Qiagen, Hilden, Germany), respectively. For cDNA synthesis, complementary DNA (cDNA) was randomly primed from $2 \mu \mathrm{g}$ of total RNA using the Omniscript reverse transcription kit (Qiagen). RT-qPCR was subsequently performed in triplicate with a 1:4 dilution of cDNA using the Quantitect SyBr green PCR system (Qiagen) on a Rotorgene 6000 series PCR machine. All primers were purchased from Qiagen. Data were collected and analyzed using $2^{-\Delta \Delta \mathrm{Ct}}$ method. Values of genes were first normalized against $\beta$-actin, and then compared to the experimental controls.

\section{Western blot}

For analysis of total protein, the protein was extracted from the lung specimens or from the cultured cells, and homogenized in RIPA lysis buffer (1\% NP40, 0.1\% SDS, $100 \mu \mathrm{g} / \mathrm{ml}$ phenylmethylsulfonyl fluoride, $0.5 \%$ sodium deoxycholate, in PBS) on ice. The supernatants were collected after centrifugation at 12000 $\times \mathrm{g}$ at $4^{\circ} \mathrm{C}$ for $20 \mathrm{~min}$. Protein concentration was determined using a BCA protein assay kit (Bio-rad, China), and whole lysates were mixed with $4 \times$ SDS loading buffer $(125 \mathrm{mmol} / \mathrm{l}$ Tris- $\mathrm{HCl}, 4 \% \mathrm{SDS}, 20 \%$ glycerol, $100 \mathrm{mmol} / \mathrm{l} \mathrm{DTT}$, and $0.2 \%$ bromophenol blue) at a ratio of $1: 3$. Protein samples were heated at $100^{\circ} \mathrm{C}$ for $5 \mathrm{~min}$ and were separated on SDS-polyacrylamide gels. The separated proteins were then transferred to a PVDF membrane. The membrane blots were first probed with a primary antibody. After incubation with horseradish peroxidase-conjugated second antibody, autoradiograms were prepared using the enhanced chemiluminescent system to visualize the protein antigen. The signals were recorded using X-ray film. Primary antibodies for Western Blot are anti- $\beta$-catenin and $\beta$-actin (all from Cell Signaling, San Jose, CA, USA). $\beta$-actin was used as protein loading controls. Secondary antibody is HRP-conjugated anti-rabbit (Jackson ImmunoResearch Labs, West Grove, PA, USA). Images shown in the figures were representative from 5 individuals. Densitometry of Western blots was quantified with NIH ImageJ software (Bethesda, MA, USA). The protein levels were first normalized to $\beta$-actin, and then normalized to experimental controls.

\section{MicroRNA target prediction and Luciferase-reporter activity assay}

MiRNAs targets were predicted as has been described before, using the algorithms TargetSan (https:// www.targetscan.org) [28]. Luciferase-reporters were successfully constructed using molecular cloning technology. Target sequence was inserted into pGL3-Basic vector (Promega) to obtain pGL3- $\beta$-catenin-3'UTR containing the miR-3619-5p binding sequence ( $\beta$-catenin-3'-UTR sequence). MiR-3619-5p-modified NSCLC cells were seeded in 24-well plates for 24 hours, after which they were transfected with $1 \mu \mathrm{g}$ of Luciferase-reporter plasmids per well using PEI Transfection Reagent. Then luciferase activities were measured using the dual-luciferase reporter gene assay kit (Promega), according to the manufacturer's instructions.

\section{Transwell cell migration assay}

The transwell cell migration assay was performed using a Fluorometric Cell Migration Assay kit with polycarbonate membrane inserts (5- $\mu \mathrm{m}$ pore size; Cell Biolabs, San Diego, CA, USA). Cells were serumstarved overnight in DMEM prior to initiation of the experiment. Cells were then incubated at $37^{\circ} \mathrm{C}$ for 24 
hours to allow cell migration through the membrane. Migratory cells were detached from the underside of the membrane and subsequently lysed and detected by CyQuant GR dye (Invitrogen). Fluorescence measurement was performed in a FluoStar Optima fluorescence plate reader with a 485 / $520 \mathrm{~nm}$ filter set.

Statistics

All statistical analyses were carried out using the SPSS 18.0 statistical software package. All values in cell and animal studies are depicted as mean \pm standard deviation and are considered significant if $p<$ 0.05. All data were statistically analyzed using one-way ANOVA with a Bonferroni correction, followed by Fisher' Exact Test for comparison of two groups. Bivariate correlations were calculated by Spearman's rank correlation coefficients.

\section{Results}

MiR-3619-5p and $\beta$-catenin levels inversely correlate in NSCLC specimens

We studied the levels of $\beta$-catenin in NSCLC specimens and detected significantly higher levels of $\beta$-catenin in NSCLC, compared to the paired adjacent non-tumor lung tissue (NLT, Fig. 1A). Moreover, we found that NSCLC expressed significantly lower levels of miR-3619$5 p$, compared to NLT (Fig. 1B). To figure out, whether this is a possible relationship between miR-3619-5p and $\beta$-catenin levels, we performed a correlation test in the resected NSCLC from 25 patients. A strong inverse correlation was detected between miR-3619-5p and $\beta$-catenin (Fig. 1C, $\gamma=-0.82 ; p<0.0001$ ), suggesting the presence of a regulatory relationship between miR-3619-5p and $\beta$-catenin in NSCLC cells.

MiR-3619-5p targets 3'-UTR of $\beta$-catenin mRNA to inhibit its expression in NSCLC cells

We then performed bioinformatics analysis, and we found that miR-3619-5p bound to 3'-UTR of $\beta$-catenin mRNA at 723th-729th base site (Fig. 2A). Then we used 2 NSCLC lines, A549 and H460, to examine whether this binding is functional. We transfected A549

Fig. 1. MiR-3619-5p and $\beta$-catenin levels inversely correlate in NSCLC specimens. (A-B) Compared with the paired non-tumor lung tissue (NLT), NSCLC expressed significantly higher levels of $\beta$-catenin by Western blot (A), and significantly lower levels of miR3619-5p by RT-qPCR (B). (C) A correlation test in the resected NSCLC specimens from 25 patients showing a strong inverse correlation between miR3619-5p and $\beta$-catenin $(\gamma=-0.82 ; p<0.0001)$. $* \mathrm{p}<0.05 . \mathrm{N}=25$.
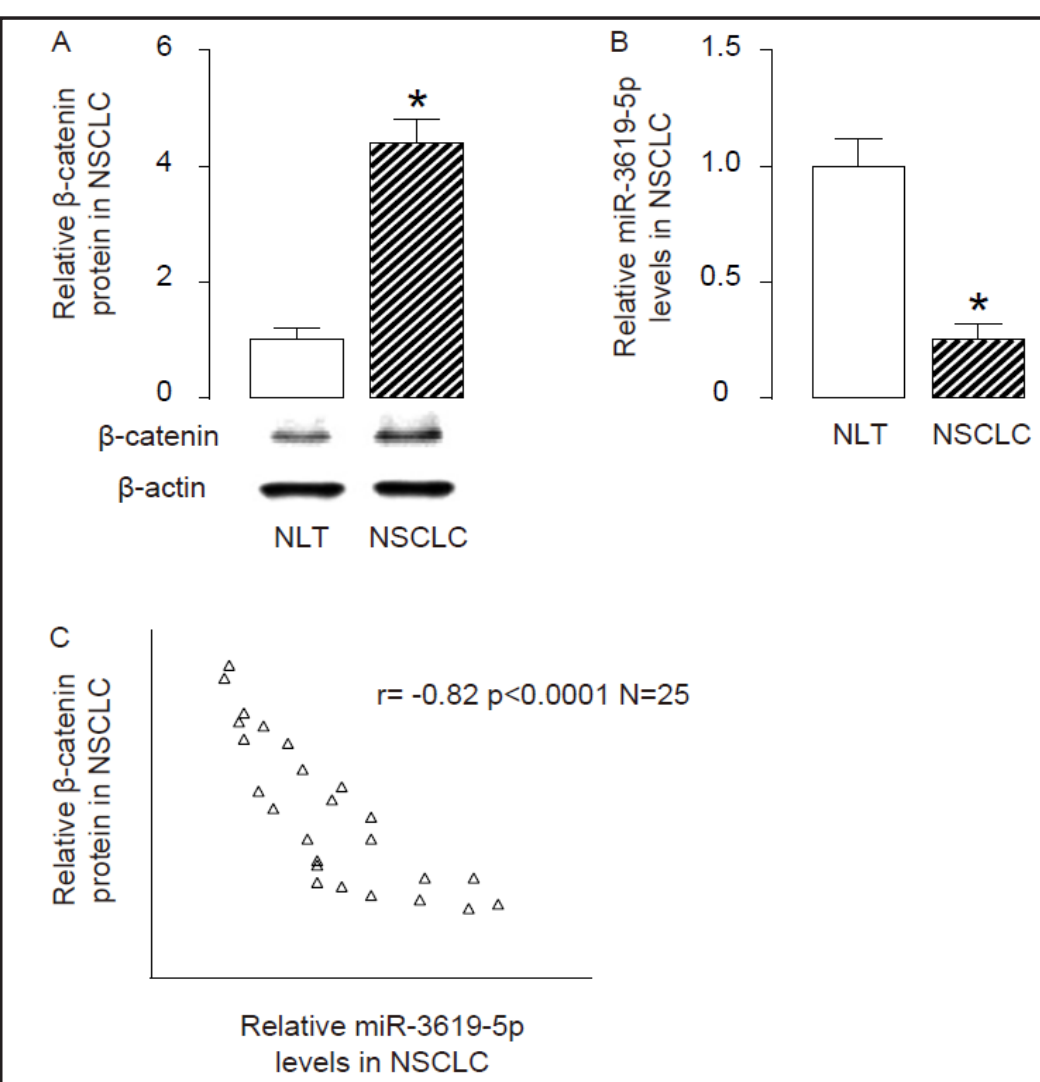


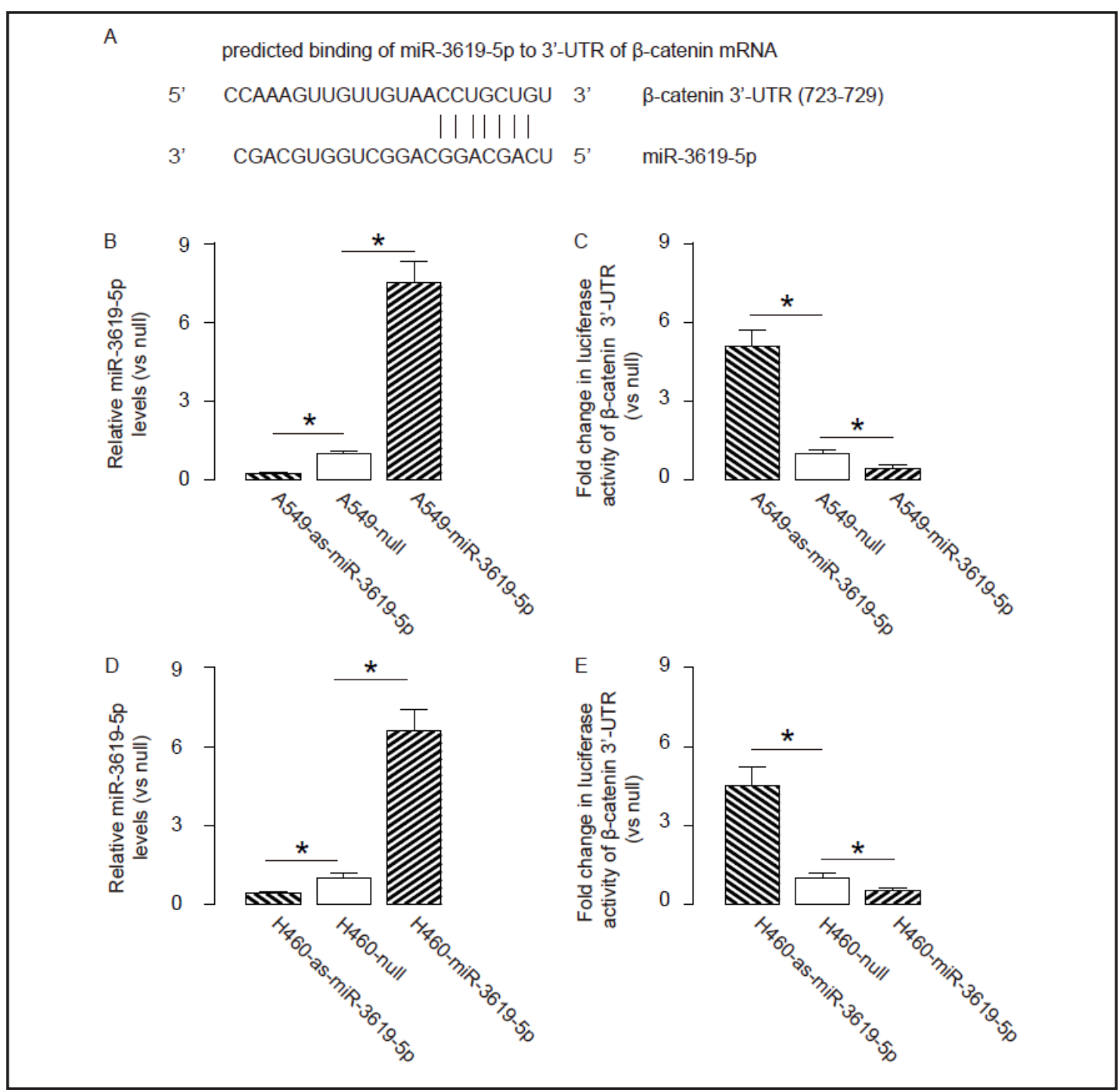

Fig. 2. MiR-3619-5p targets 3'-UTR of $\beta$-catenin mRNA to inhibit its expression in NSCLC cells. (A) Bioinformatics analysis of $\beta$-catenin targeting miRNAs, showing that miR-3619-5p binds to 3'-UTR of $\beta$-catenin mRNA at 723th-729th base site. (B) A549 cells were transfected with plasmids carrying either miR-36195p, or as-miR-3619-5p, or null as a control. The modification of miR-3619-5p levels in A549 cells was confirmed by RT-qPCR. (C) The miR-3619-5p-modified A549 cells were transfected with $1 \mu \mathrm{g}$ of $\beta$-catenin-3'-UTR Luciferase-reporter plasmid and the luciferase activities were examined. (D) H460 cells were transfected with plasmids carrying either miR-3619-5p, or as-miR-3619-5p, or null as a control. The modification of miR-3619-5p levels in H460 cells was confirmed by RT-qPCR. (C) The miR-3619-5p-modified H460 cells were transfected with $1 \mu \mathrm{g}$ of $\beta$-catenin-3'-UTR Luciferase-reporter plasmid and the luciferase activities were examined. ${ }^{*} \mathrm{p}<0.05 . \mathrm{N}=5$.

and H460 cells with plasmids carrying either miR-3619-5p, or as-miR-3619-5p, or null as a control. The modification of miR-3619-5p levels in A549 cells was confirmed by RTqPCR (Fig. 2B). Then, these cells were transfected with $1 \mu \mathrm{g}$ of $\beta$-catenin-3'-UTR Luciferasereporter plasmid. We found that the luciferase activities in A549-as-miR-3619-5p cells were significantly higher than the control, while the luciferase activities in A549-miR-3619-5p cells were significantly lower than the control (Fig. 2C). Similarly, the modification of miR3619-5p levels in H460 cells was confirmed by RT-qPCR (Fig. 2D). Then, these cells were transfected with $1 \mu \mathrm{g}$ of $\beta$-catenin-3'-UTR Luciferase-reporter plasmid. We found that the luciferase activities in H460-as-miR-3619-5p cells were significantly higher than the control, 
Fig. 3. MiR-36195p suppresses $\beta$-catenin protein, without affecting $\beta$-catenin mRNA in NSCLC cells. (A) The mRNA levels of $\beta$-catenin in miR-36195p-modified A549 cells by RT-qPCR. (B) The protein levels of $\beta$-catenin in miR3619-5p-modified A549 cells by Western blot. (C) The mRNA levels of $\beta$-catenin in miR-36195p-modified H460 cells by RT-qPCR. (D) The protein levels of $\beta$-catenin in miR3619-5p-modified H460 cells by Western blot. ${ }^{*} \mathrm{p}<0.05$. NS: non-significant. $\mathrm{N}=5$.
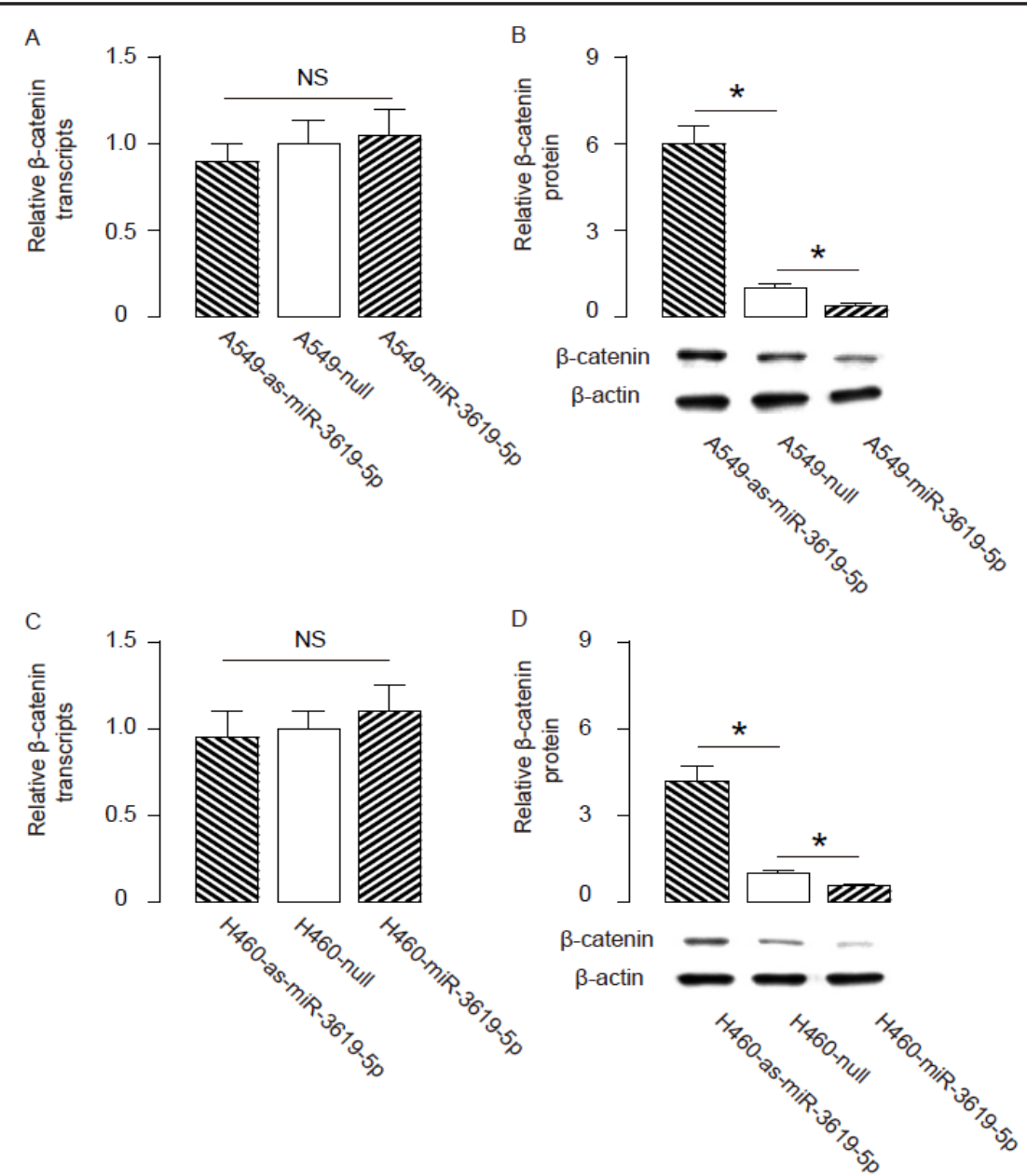

Fig. 4. MiR-3619-5p inhibits NSCLC cell growth. (AB) MTT assay for miR-3619-5p-modified A549 cells (A), and H460 cells (B). * ${ }^{*}<0.05 . \mathrm{N}=5$.

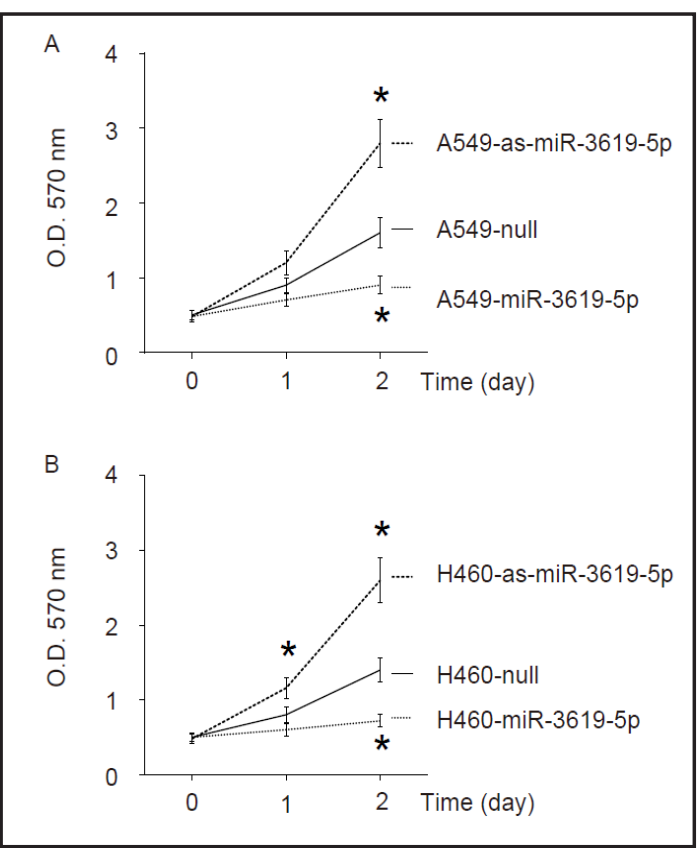

while the luciferase activities in H460-miR-3619-5p cells were significantly lower than the control (Fig. 2E). Together, these data demonstrate that miR-3619-5p targets 3'-UTR of 


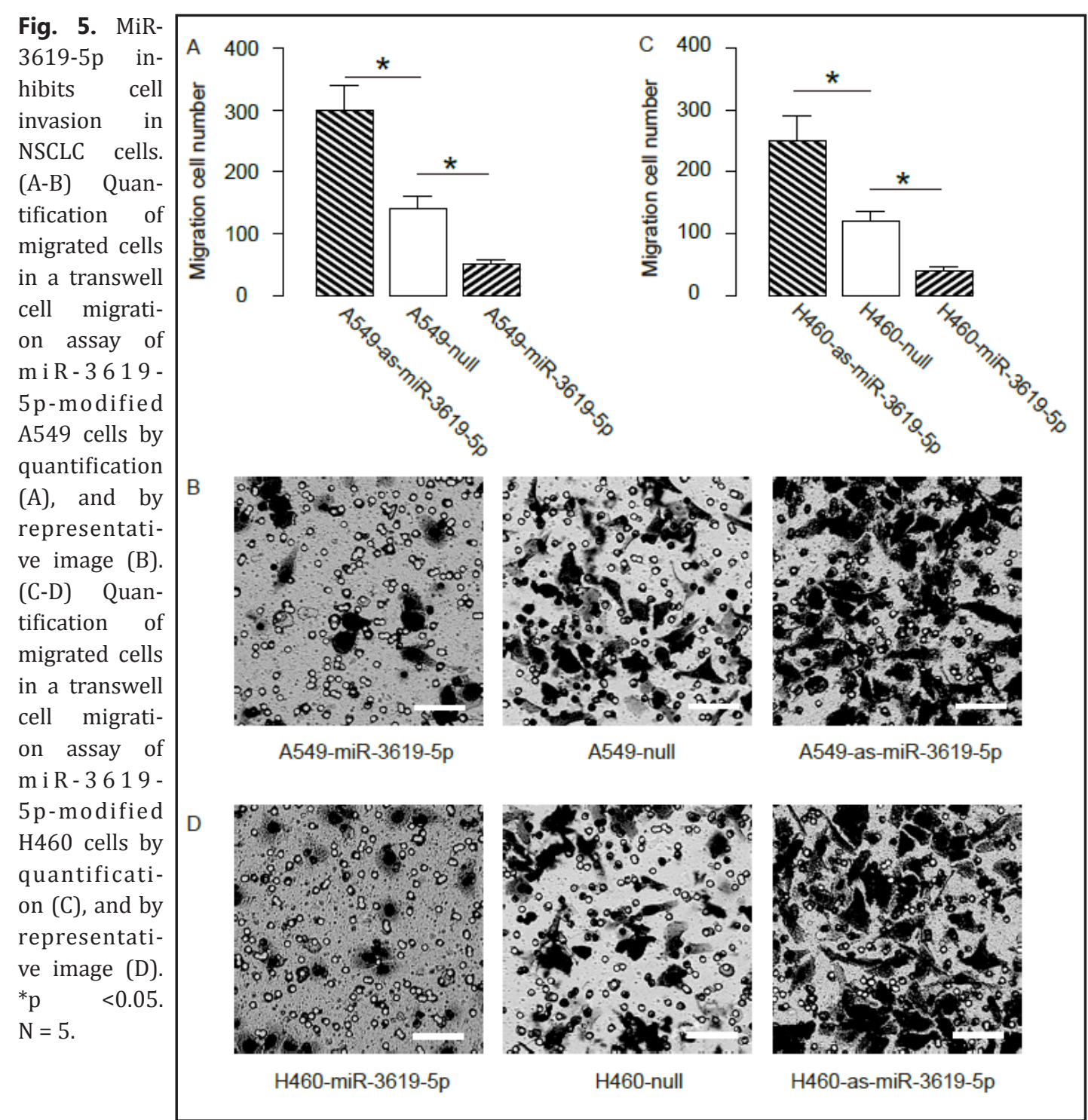

$\beta$-catenin to inhibit its translation in NSCLC cells, and loss of miR-3619-5p in NSCLC may result in increases in $\beta$-catenin, or augmented WNT signaling, in NSCLC cells.

MiR-3619-5p suppresses $\beta$-catenin protein, without affecting $\beta$-catenin mRNA in NSCLC cells

Moreover, we found that although the mRNA levels of $\beta$-catenin in A549 cells were unchanged by miR-3619-5p modification (Fig. 3A), overexpression of miR-3619-5p in A549 cells decreased $\beta$-catenin protein, while depletion of miR-3619-5p in A549 cells increased $\beta$-catenin protein (Fig. 3B). Similarly, although the mRNA levels of $\beta$-catenin in H460 cells were unchanged by miR-3619-5p modification (Fig. 3C), overexpression of miR-3619-5p in $\mathrm{H} 460$ cells decreased $\beta$-catenin protein, while depletion of miR-3619-5p in H460 cells increased $\beta$-catenin protein (Fig. 3D). Together, these data suggest that miR-3619-5p suppresses $\beta$-catenin protein, without affecting $\beta$-catenin transcript levels in NSCLC cells.

MiR-3619-5p inhibits NSCLC cell growth

In an MTT assay, we found that overexpression of miR-3619-5p in A549 cells resulted in a significant decrease in cell growth, while depletion of miR-3619-5p in A549 cells resulted in a significant increase in cell growth (Fig. 4A). Similarly, overexpression of miR-3619-5p in 


\section{Cellular Physiology Cell Physiol Biochem 2015;37:1527-1536

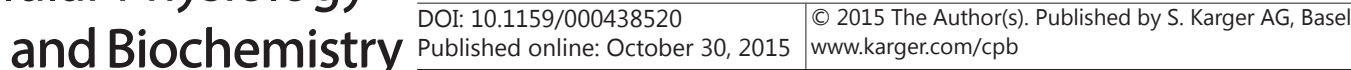 \\ Niu/Liu/Jia/Chen: MiR-3619-5p Regulates NSCLC Growth and Invasion}

Fig. 6. A schematic model. MiR-3619-5p targets 3'-UTR of $\beta$-catenin mRNA to inhibit its translation in NSCLC cells. Loss of miR-3619-5p in NSCLC promotes cancer cell growth and invasion through augmenting $\beta$-catenin.

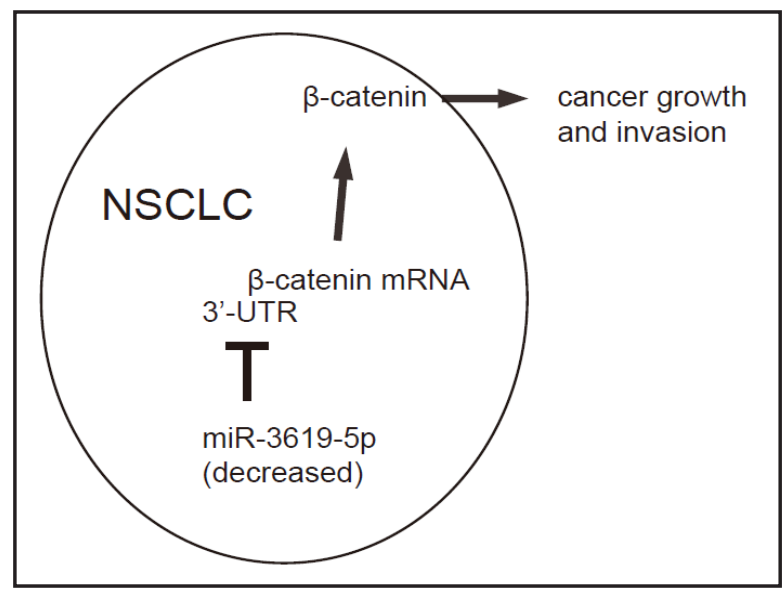

H460 cells resulted in a significant decrease in cell growth, while depletion of miR-3619-5p in H460 cells resulted in a significant increase in cell growth (Fig. 4B). Together, these data suggest that miR-3619-5p inhibits NSCLC cell growth.

MiR-3619-5p inhibits cell invasion in NSCLC cells

Next, we evaluated the effects of miR-3619-5p on NSCLC cell invasiveness. We found that overexpression of miR-3619-5p in A549 cells resulted in a significant decrease in cell invasion, while depletion of miR-3619-5p in A549 cells resulted in a significant increase in cell invasion, in a transwell cell migration assay, by quantification (Fig. 5A), and by representative images (Fig. 5B). Similarly, overexpression of miR-3619-5p in H460 cells resulted in a significant decrease in cell invasion, while depletion of miR-3619-5p in H460 cells resulted in a significant increase in cell invasion, in a transwell cell migration assay, by quantification (Fig. 5C), and by representative images (Fig. 5D). Together, these data suggest that miR-3619-5p inhibits cell invasion in NSCLC cells. To summarize, our data demonstrate a previously unappreciated role of miR-3619-5p in suppressing $\beta$-catenin-mediated cancer vascularization, growth and invasion in NSCLC (Fig. 6).

\section{Discussion}

NSCLC is a malignant lung cancer that affects millions of people worldwide [3, 5-7]. It is one of the most common causes of cancer-associated death [3, 5-7]. The poor outcome of NSCLC largely results from its fast growth and invasion [3, 5-7]. Here, we focused on the miRNA-regulated WNT signaling in regulation of the growth and invasion of NSCLC.

Generally, the protein regulation includes regulation of protein degradation by different protein modifications, e.g. phosphorylation, SUMOylation, acetylation and ubiquitination, and regulation of protein translation by miRNAs. In this study, we focused on miRNAs. First, we compared the levels of $\beta$-catenin and some candidate miRNAs that target $\beta$-catenin in NSCLC specimens, and we specifically found that miR-3619-5p inversely correlated the levels of $\beta$-catenin in NSCLC tissue. Thus, we hypothesized that in NSCLC, $\beta$-catenin may be regulated by miR-3619-5p at post-transcriptional level. Based on bioinformatics analyses and promoter reporter assay, we found that the binding of miR-3619-5 to $\beta$-catenin mRNA is functional, which was further supported by the examination of $\beta$-catenin mRNA and protein levels in miR-3619-5p-modified NSCLC cell lines.

Furthermore, miR-3619-5p overexpression resulted in suppression of NSCLC cell growth and invasion, while miR-3619-5p depletion increased NSCLC growth and invasion. Hence, the significant reduction of miR-319-5p levels in NSCLC specimens has significance in that it allows activation of WNT/ $\beta$-catenin signaling, which directly contributes to the cancer growth and metastases. 


\section{Cellular Physiology Cell Physiol Biochem 2015;37:1527-1536

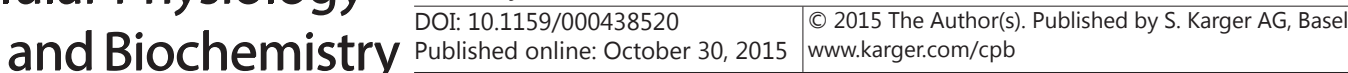 \\ Niu/Liu/Jia/Chen: MiR-3619-5p Regulates NSCLC Growth and Invasion}

Future approaches may be performed to improve our understanding of the regulation of miR-3619-5p in NSCLC and to determine how miR-3619-5p is suppressed in NSCLC. On the other hand, it is possible that miR-3619-5p may have other targets that are related to either cancer cell growth or cell invasion in NSCLC cells. Elucidation of targets of miR-3619$5 p$ other than $\beta$-catenin may improve the completeness of our understanding of the role of miR-3619-5p in the carcinogenesis of NSCLC.

Here, we have shown similar data on two NSCLC cell lines and got essentially same results. These approaches thus ruled out a possibility of our findings to be cell-line dependent. In summary, our data demonstrate a previously unappreciated role of miR-3619$5 \mathrm{p}$ in controlling of $\beta$-catenin-associated cancer vascularization, growth and invasion, and this regulation is conducted through modulation of $\beta$-catenin protein translation.

\section{Disclosure Statement}

The authors have declared that no competing interests exist.

\section{References}

1 Caramori G, Casolari P, Cavallesco GN, Giuffre S, Adcock I, Papi A: Mechanisms involved in lung cancer development in copd. Int J Biochem Cell Biol 2011;43:1030-1044.

2 Buttery RC, Rintoul RC, Sethi T: Small cell lung cancer: The importance of the extracellular matrix. Int J Biochem Cell Biol 2004;36:1154-1160.

3 Pei J, Lou Y, Zhong R, Han B: Mmp9 activation triggered by epidermal growth factor induced foxo1 nuclear exclusion in non-small cell lung cancer. Tumour Biol 2014;35:6673-6678.

4 Hu X, Zhang F, Liu XR, Wu YT, Ni YM: Efficacy and potential microrna mechanism for computed tomography-guided percutaneous radiofrequency ablation of primary lung cancer and lung metastasis from liver cancer. Cell Physiol Biochem 2014;33:1261-1271.

5 Ji Y, Gao F, Sun B, Hao J, Liu Z: Angiotensin-converting enzyme 2 inhibits apoptosis of pulmonary endothelial cells during acute lung injury through suppressing smad2 phosphorylation. Cell Physiol Biochem 2015;35:2203-2212.

6 Jian H, Zhao Y, Liu B, Lu S: Sema4b inhibits growth of non-small cell lung cancer in vitro and in vivo. Cell Signal 2015;27:1208-1213.

7 Jian H, Zhao Y, Liu B, Lu S: Sema4b inhibits mmp9 to prevent metastasis of non-small cell lung cancer. Tumour Biol 2014;35:11051-11056.

8 Tang Q, Zhao S, Wu J, Zheng F, Yang L, Hu J, Hann SS: Inhibition of integrin-linked kinase expression by emodin through crosstalk of ampkalpha and erk1/2 signaling and reciprocal interplay of sp1 and c-jun. Cell Signal 2015;27:1469-1477.

9 Hahn SS, Tang Q, Zheng F, Zhao S, Wu J: Gw1929 inhibits alpha7 nachr expression through ppargammaindependent activation of p38 mapk and inactivation of pi3-k/mtor: The role of egr-1. Cell Signal 2014;26:730-739.

10 Zhao L, Wang DL, Liu Y, Chen S, Sun FL: Histone acetyltransferase hmof promotes s phase entry and tumorigenesis in lung cancer. Cell Signal 2013;25:1689-1698.

11 Xie C, Jiang G, Fan C, Zhang X, Zhang Y, Miao Y, Lin X, Wu J, Wang L, Liu Y, Yu J, Yang L, Zhang D, Xu K, Wang E: Armc8alpha promotes proliferation and invasion of non-small cell lung cancer cells by activating the canonical wnt signaling pathway. Tumour Biol 2014;35:8903-8911.

12 Stewart DJ: Wnt signaling pathway in non-small cell lung cancer. J Natl Cancer Inst 2014;106:djt356.

13 Winn RA, Van Scoyk M, Hammond M, Rodriguez K, Crossno JT, Jr., Heasley LE, Nemenoff RA: Antitumorigenic effect of wnt 7a and fzd 9 in non-small cell lung cancer cells is mediated through erk-5dependent activation of peroxisome proliferator-activated receptor gamma. J Biol Chem 2006;281:2694326950. 


\section{Cellular Physiology Cell Physiol Biochem 2015;37:1527-1536

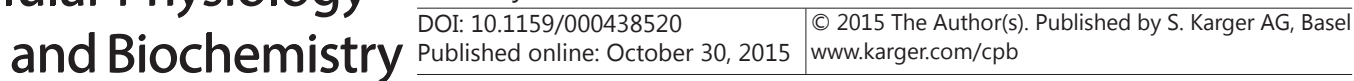 \\ Niu/Liu/Jia/Chen: MiR-3619-5p Regulates NSCLC Growth and Invasion}

14 Kren L, Hermanova M, Goncharuk VN, Kaur P, Ross JS, Pavlovsky Z, Dvorak K: Downregulation of plasma membrane expression/cytoplasmic accumulation of beta-catenin predicts shortened survival in non-small cell lung cancer. A clinicopathologic study of 100 cases. Cesk Patol 2003;39:17-20.

15 He B, You L, Uematsu K, Xu Z, Lee AY, Matsangou M, McCormick F, Jablons DM: A monoclonal antibody against wnt-1 induces apoptosis in human cancer cells. Neoplasia 2004;6:7-14.

16 Akiri G, Cherian MM, Vijayakumar S, Liu G, Bafico A, Aaronson SA: Wnt pathway aberrations including autocrine wnt activation occur at high frequency in human non-small-cell lung carcinoma. Oncogene 2009;28:2163-2172.

17 Lee JS, Hur MW, Lee SK, Choi WI, Kwon YG, Yun CO: A novel slrp6e1e2 inhibits canonical wnt signaling, epithelial-to-mesenchymal transition, and induces mitochondria-dependent apoptosis in lung cancer. PLoS One 2012;7:e36520.

18 Mei Q, Li F, Quan H, Liu Y, Xu H: Busulfan inhibits growth of human osteosarcoma through mir-200 family micrornas in vitro and in vivo. Cancer Sci 2014;105:755-762.

19 Wang F, Xiao W, Sun J, Han D, Zhu Y: Mirna-181c inhibits egfr-signaling-dependent mmp9 activation via suppressing akt phosphorylation in glioblastoma. Tumour Biol 2014;35:8653-8658.

20 Liu G, Jiang C, Li D, Wang R, Wang W: Mirna-34a inhibits egfr-signaling-dependent mmp7 activation in gastric cancer. Tumour Biol 2014;35:9801-9806.

21 Chang Y, Zhao Y, Gu W, Cao Y, Wang S, Pang J, Shi Y: Bufalin inhibits the differentiation and proliferation of cancer stem cells derived from primary osteosarcoma cells through mir-148a. Cell Physiol Biochem 2015;36:1186-1196.

22 Ji D, Li B, Shao Q Li F, Li Z, Chen G: Mir-22 suppresses bmp7 in the development of cirrhosis. Cell Physiol Biochem 2015;36:1026-1036.

23 Song W, Li Q, Wang L, Wang L: Modulation of foxo1 expression by mir-21 to promote growth of pancreatic ductal adenocarcinoma. Cell Physiol Biochem 2015;35:184-190.

24 Zhang L, Qian J, Qiang Y, Huang H, Wang C, Li D, Xu B: Down-regulation of mir-4500 promoted non-small cell lung cancer growth. Cell Physiol Biochem 2014;34:1166-1174.

25 Wu N, Zhang C, Bai C, Han YP, Li Q: Mir-4782-3p inhibited non-small cell lung cancer growth via usp14. Cell Physiol Biochem 2014;33:457-467.

26 Giard DJ, Aaronson SA, Todaro GJ, Arnstein P, Kersey JH, Dosik H, Parks WP: In vitro cultivation of human tumors: Establishment of cell lines derived from a series of solid tumors. J Natl Cancer Inst 1973;51:14171423.

27 Banks-Schlegel SP, Gazdar AF, Harris CC: Intermediate filament and cross-linked envelope expression in human lung tumor cell lines. Cancer Res 1985;45:1187-1197.

28 Coronnello C, Benos PV: Comir: Combinatorial microrna target prediction tool. Nucleic Acids Res 2013;41:W159-164. 